\title{
PRÁTICAS DO PMBOK® APLICADAS A ÁREA DE DESENVOLVIMENTO DE PROCESSOS *
}

\author{
Henrique Dias Gatti Turrer ${ }^{1}$ \\ Carlos Magno Nascimento ${ }^{1}$ \\ Jose Francisco Cabello Russo ${ }^{1}$ \\ Henrique Lopes Martins Pereira ${ }^{1}$ \\ Douglas Batista Mazzinghy²
}

\section{Resumo}

Atividades de pesquisa e desenvolvimento (P\&D) possuem efeito positivo nos indicadores econômico-financeiros das organizações. Essas atividades possuem características de projetos, e, por isso, podem ser submetidas aos processos de gerenciamento recomendados pelo PMBOK. As atividades da gerência de P\&D do Minas-Rio da Anglo American foram submetidas à alguns desses processos. Inicialmente, foram estratificados os processos por magnitude de esforço e de impacto. Oito processos foram classificados como de baixo esforço e alto impacto e, por isso, totalmente implementados. A criação e aplicação de três ativos organizacionais padronizados para todas as atividades realizadas permitiu 0 desenvolvimento de grande parte dos processos considerados mais importantes. Um questionário de avaliação do grau de maturidade em gerenciamento de projetos foi respondido por parte da equipe antes e depois da adoção formal de tais processos. O grau de maturidade aumentou significativamente, $150 \%$, em dois anos, passando do nível conceitual para padronizado. Além disso, foi observada um aumento significativo, igual a $731 \%$, no número de atividades realizadas pela área.

Palavras-chave: Gerenciamento de projetos; laboratório de pesquisa e desenvolvimento; PMBOK.

\section{PMBOK@'S RECOMENDATIONS APPLIED TO RESEARCH AND DEVELPMENT AREA}

\section{Abstract}

Research and development (R\&D) activities have a positive effect on the organizations' key parameters indicator (KPI). These activities also have project characteristics, so they might be submitted to the processes suggested by PMBOK. The R\&D activities performed by R\&D management of Anglo America's Minas-Rio adopted some of these processes. Initially, the processes were stratified by effort and impact magnitude. Eight processes were classified as low effort and high impact and, thus, totally implemented. The creation and application of three standardized organizational assets for all activities enabled the development of the most important processes. A questionnaire for the determination of project managing maturity level was answered by some part of the management's team before and after the formal use of those processes. In two years, the maturity level has increased significantly, $150 \%$, going from the conceptual level to the standardized one. Furthermore, a significant increase in the amount of activities produced by the area was observed, meaning a $731 \%$ improvement.

Keywords: Project management; research and development lab; PMBOK. 


\section{INTRODUÇÃO}

A gerência de desenvolvimento de processos de uma mineração é, usualmente, a área responsável por atividades de pesquisa (P\&D) relativas ao processo produtivo da empresa. Em geral, a maioria das atividades realizadas não são consideradas rotineiras, ou seja, possuem características de projetos. Apesar do baixo custo que tais atividades possuem, em relação ao montante investido pela organização anualmente, as mesmas tem efeito significativo em melhorias que impactam a margem de lucro. Por isso, a aplicação de técnicas de gerenciamento de projetos pode constituir uma importante ferramenta para o sucesso dessas atividades e, consequentemente, dos objetivos estratégicos da organização.

O Minas-Rio é uma operação que possui capacidade para produção de 26,5 milhões de toneladas naturais por ano de pellet feed, extraído da jazida da Serra do Sapo em Conceição do Mato Dentro, MG, processado por um concentrador na mesma localidade e bombeado por 529 km de mineroduto até São João da Barra, RJ, onde é embarcado.

A grande maioria das atividades desenvolvidas pela área possuem pequena duração, de alguns dias a poucas semanas. É comum algumas atividades serem recorrentes, como num ciclo de melhoria continua. Normalmente são conduzidas com recursos da própria gerência. O orçamento anual da área é ajustado a cada ano. Apesar do valor pouco significativo para a organização, menor que $1 \%$ do custo total, o efeito das atividades da área possui grande relevância. Por isso, qualquer esforço no sentido de aumentar seu sucesso tem impacto significativo para a organização.

Exemplos de atividades desenvolvidas pela área da empresa estão disponíveis em anais e periódicos especializados (CRUZ, 2010; TURRER et al., 2013a; TURRER et al., 2013b; MAZZINGHY et al., 2013; MAZZINGHY et al., 2014; TURRER et al., 2015; ESTEVES et al., 2016; BENZER et al., 2017; MAZZINGHY et al., 2017).

Segundo o PMBOK (PMI, 2013), um projeto é um esforço temporário empreendido para criar um produto, serviço ou resultado exclusivo. Logo, as atividades desenvolvidas pela área de gerenciamento de processos do Minas-Rio da Anglo American podem ser caracterizadas como projetos.

O objetivo principal deste trabalho foi identificar ferramentas e boas práticas recomendadas pelo $\mathrm{PMBOK}$ que são aplicáveis às atividades da área de desenvolvimento de processos e verificar sua eficácia através de suas utilizações.

\section{MATERIAIS E MÉTODOS}

Inicialmente, foi feita uma pesquisa documental que consistiu do mapeamento das ferramentas recomendadas no PMOK (PMI, 2013). Em seguida, essas ferramentas foram priorizadas utilizando-se um matriz impacto versus esforço.

Avaliação da efetividade dos processos desenvolvidos foi realizada através de respostas à um questionário de grau de maturidade em gerenciamento de projetos da área. O questionário foi respondido por $46 \%$ do efetivo da área em dois momentos distintos, antes da consolidação dos processos na área, o que ocorreu entre 2013 e 2014, e após, em setembro de 2015.

O questionário adotado foi baseado principalmente naquele proposto por NETO (2011). Algumas perguntas, que não apresentavam uma aplicação relevante para o caso estudado, foram excluídas. A cada pergunta foi atribuída uma nota de 0 a 7 , sendo que 0 significa a total discordância. Quanto maior o número, maior o grau de 
concordância. A mediana de cada aspecto foi usada para determinação do grau de maturidade, que possuía 7 níveis diferentes conforme tabela 1.

Tabela 1. Nível de maturidade e nota

\begin{tabular}{llllllll}
\hline Resultado & $0>x>1$ & $1 \geq x>2$ & $2 \geq x>3$ & $3 \geq x>4$ & $4 \geq x>5$ & $5 \geq x>6$ & $x \geq 6$ \\
\hline \multirow{2}{*}{ Maturidade } & $\begin{array}{l}\text { Inexisten- } \\
\text { te }\end{array}$ & $\begin{array}{l}\text { Concei- } \\
\text { tual }\end{array}$ & $\begin{array}{l}\text { Programa- } \\
\text { do }\end{array}$ & $\begin{array}{l}\text { Padroniza- } \\
\text { do }\end{array}$ & $\begin{array}{l}\text { Contrata- } \\
\text { do }\end{array}$ & $\begin{array}{l}\text { Implanta- } \\
\text { do }\end{array}$ & $\begin{array}{l}\text { Estraté- } \\
\text { gico }\end{array}$ \\
\hline
\end{tabular}

\section{RESULTADOS E DISCUSSÃO}

Os processos foram estratificados numa matriz de esforço versus impacto, conforme figura 1.

Apesar da predominância de processos no quadrante III, de baixo esforço e baixo impacto, os mesmos apresentaram uma distribuição relativamente equilibrada nos quatro quadrantes. O quadrante I, alto impacto e baixo esforço, ficou com $21 \%$ dos processos, ou seja, oito. Julgou-se essa quantidade como suficiente para promover o amadurecimento inicial almejado pela área e, por isso, os processos localizados ali foram os únicos desenvolvidos com iniciativas formais e estruturadas.

Para o processo de desenvolver do termo de abertura do projeto, foi adotada a utilização/preenchimento de um documento que contém algumas informações básicas, como: número da solicitação, dados do requisitante usadas, principalmente, nas comunicações que se fazem necessárias durante e após a realização das atividades, nome do projeto, objetivo, local para disponibilização das informações relativas à atividade e, comumente, orientações para os executantes das atividades. Sem a formalização desse importante processo, era comum que atividades fossem iniciadas sem o conhecimento de todos os membros da equipe, impactando o prazo ou até qualidade de atividades que haviam sido planejadas. Era comum, por exemplo, que funcionários de outras áreas, inclusive gerentes funcionais, requisitassem diretamente para membros da área a execução de atividades que não haviam sido planejadas e, muitas vezes, nem eram do conhecimento de toda equipe. Isso causava um descontrole na programação das atividades, impactando negativamente o sucesso de várias delas.

O processo de definir as atividades é realizado através do preenchimento dos documentos criados. O primeiro documento é o plano de trabalho discutido anteriormente, que possui um campo para descrição das atividades. O outro documento é o cronograma de controle. Não existe uma padronização para o nível de detalhamento das atividades. Em geral, projetos mais complexos, que englobam um maior número de atividades, que demandam um tempo maior para execução e/ou representam atividades com as quais a equipe possui menor experiência, são mais bem descritos. Sem a formalização desse processo, era comum atividades começarem e mudarem várias vezes durante sua execução, podendo se estender por tempo indeterminado.

Para o processo de estimar as durações das atividades, a opinião especializada e técnicas de tomada de decisão em grupo são as mais usadas na gerência. Em alguns casos, estimativa análoga também é usada devido às semelhanças entre alguns dos projetos. Como grande parte das atividades da área possui um pequeno período de execução, a maioria não tinha seu cronograma registrado. A pesar do efeito pequeno de cada uma, as mesmas somadas acabavam impactando o sucesso de outros projetos maiores. Antes da formalização desse processo, não se tinha um prazo de término de cada atividade. Logo, as previsões para relocação de 
recursos eram pouco precisas, o que comprometia as expectativas de início e fim de outras atividades.

\begin{tabular}{|c|c|c|c|}
\hline \multirow{2}{*}{ 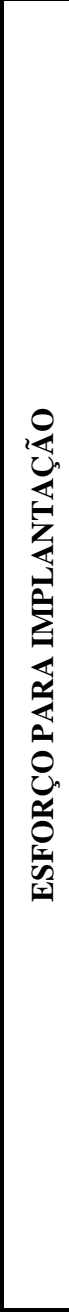 } & $\stackrel{0}{2}$ & $\begin{array}{l}\text { Coletar os requisitos (P / esc.) } \\
\text { Controlar a qualidade (M\&C / qual.) } \\
\text { Controlar o escopo (M\&C / esc.) } \\
\text { Controlar os custos (M\&C / cust.) } \\
\text { Desenvolver o cronograma (P / temp.) } \\
\text { Planejar o gerenciamento das comunicações (P / com.) } \\
\text { Planejar o gerenciamento das partes interessadas (P / } \\
\text { p.i) } \\
\text { Planejar o gerenciamento do cronograma (P / temp.) } \\
\text { Planejar o gerenciamento do escopo (P / esc.) } \\
\text { Planejar o gerenciamento dos recursos humanos (P / } \\
\text { r.h.) } \\
\text { Realizar a análise quantitativa dos riscos (P / risc.) } \\
\text { Realizar a garantia da qualidade (EX / qual.) } \\
\text { Realizar o controle integrado de mudanças (M\&C / int.) }\end{array}$ & $\begin{array}{l}\text { Criar a estrutura analítica do projeto, EAP (P / esc.) } \\
\text { Controlar o engajamento das partes interessadas (M\&C / } \\
\text { p.i) } \\
\text { Controlar os riscos (M\&C / risc.) } \\
\text { Desenvolver o plano de gerenciamento do projeto (P / } \\
\text { int.) } \\
\text { Estimar os recursos das atividades (P / temp.) } \\
\text { Identificar os riscos ( } \mathrm{P} / \text { risc.) } \\
\text { Orientar e gerenciar o trabalho do projeto (EX / int.) } \\
\text { Planejar as respostas aos riscos (P / risc.) } \\
\text { Planejar o gerenciamento da qualidade (P / qual.) } \\
\text { Planejar o gerenciamento dos riscos (P / risc.) } \\
\text { Realiar a análise qualitativa dos riscos (P / risc.) }\end{array}$ \\
\hline & 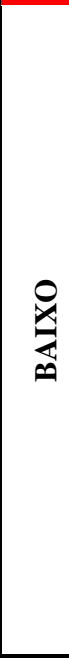 & $\begin{array}{l}\text { Conduzir as aquisições (EX / aqu.) } \\
\text { Controlar as aquisições (M\&C / aqu.) } \\
\text { Controlar as comunicações (M\&C / com.) } \\
\text { Definir o escopo (P / esc.) } \\
\text { Determinar o orçamento (P / cust.) } \\
\text { Encerrar as aquisições (EN / aqu.) } \\
\text { Estimar os custos (P / cust.) } \\
\text { Gerenciar as comunicações (EX / com.) } \\
\text { Gerenciar o engajamento das partes interessadas (EX / } \\
\text { p.i) } \\
\text { Identificar as partes interessadas (I / p.i) } \\
\text { Mobilizar a equipe do projeto (EX / r.h.) } \\
\text { Planejar o gerenciamento das aquisições (P / aqu.) } \\
\text { Planejar o gerenciamento dos custos (P / cust.) } \\
\text { Sequenciar as atividades (P / temp.) } \\
\text { Validar o escopo (M\&C / esc.) }\end{array}$ & $\begin{array}{l}\text { Controlar o cronograma (M\&C / temp.) } \\
\text { Definir as atividades (P / temp.) } \\
\text { Desenvolver a equipe do projeto (EX / r.h.) } \\
\text { Desenvolver o termo de abertura do projeto (I / int.) } \\
\text { Encerrar o projeto ou fase (EN / int.) } \\
\text { Estimar as durações das atividades (P / temp.) } \\
\text { Gerenciar a equipe do projeto (EX / r.h.) } \\
\text { Monitorar e controlar o trabalho do projeto (M\&C / int.) }\end{array}$ \\
\hline & & BAIXO & ALTO \\
\hline & & IMPACTO NC & RESULTADO \\
\hline \multicolumn{4}{|c|}{ Legenda } \\
\hline & I & Iniciação & Integração \\
\hline & $\mathrm{P}$ & Planejamento & Escopo \\
\hline & EX & Execução & Tempo \\
\hline & $\begin{array}{l}\text { 1\&C } \\
\mathrm{EM}\end{array}$ & $\begin{array}{l}\text { Monitoramento e controle } \\
\text { Encerramento }\end{array}$ & $\begin{array}{l}\text { Custo } \\
\text { Qualidade } \\
\text { Recursos humanos } \\
\text { Comunicação } \\
\text { Risco } \\
\text { Aquisição } \\
\text { Partes interessadas }\end{array}$ \\
\hline
\end{tabular}

Figura 1. Priorização dos processos que terão foco para aplicação na gerência.

Processos relativos ao R.H. possuem algumas características particulares. A primeira é que já eram desenvolvidos, numa intensidade maior do que os demais processos que foram classificados no quadrante I, devido a iniciativas da área de R.H. com a gerência. A segunda é que esses processos não estão relacionados à projetos individualmente, uma vez que grande parte da equipe é composta pelo mesmo grupo de pessoas, ou seja, colaboradores da gerência. 
A nomeação de um supervisor, que ficaria responsável diretamente pelos processos de R.H. de grande parte da equipe, que inclui os técnicos e operadores, foi um catalisador para que os processos de desenvolver e gerenciar a equipe do projeto ocorressem mais eficientemente.

Comumente quando algum fator de sucesso não atende o requisito, as atividades são refeitas, de forma que no fim, prazo é o principal aspecto impactado. Logo, uma saída desse processo de desenvolver a equipe do projeto, que é constantemente praticada, é a avaliação do desempenho da equipe em relação ao prazo de execução das atividades. O feedback é fornecido rotineiramente e informalmente para os membros da equipe, como ferramenta do processo de gerenciar a equipe do projeto. Pontos positivos e de melhorias são constantemente discutidos e comunicados.

A utilização de ferramentas dos dois processos de R.H. como o uso de habilidades interpessoais e da inteligência emocional para aumentar a cooperação é facilitada, uma vez que a maioria dos membros da gerência já se conhecem e trabalham juntos há alguns anos.

$\mathrm{Na}$ grande maioria dos casos, a principal ferramenta utilizada para o processo de monitorar e controlar o trabalho do projeto são reuniões diárias e presenciais de poucos minutos entre parte dos membros da equipe, principalmente os executores e o supervisor. Reuniões informais e por telefone também são comuns. As atualizações ocorrem nos ativos organizacionais já mencionados em outros processos. As planilhas em que os resultados são registrados, além de ser um ativo organizacional que auxilia no processo de definição as atividades, também auxilia o processo de monitorar e controlar o trabalho do projeto.

Em relação ao processo de controlar o cronograma, a principal entrada utilizada é o próprio cronograma já citado anteriormente. Técnicas de compressão de cronograma são usadas comumente. O paralelismo é menos comum já que em muitos casos pode ocorrer uma interação negativa entre as atividades. Novamente, a atualização do cronograma apresenta algumas das saídas desse processo. Além de possuir informações sobre o desempenho do trabalho, apresenta uma previsão de termino.

O processo de encerrar o projeto pode ocorrer de duas formas. Quando o solicitante é de outra gerência, essa fase se inicia com o envio dos resultados de testes para o mesmo. Com os resultados, ele é, então, capaz de fazer suas análises e confirmar, ou não, a aceitação da entrega. Nesses casos a transição do produto, serviço ou resultado final é efetuado nesse momento. Contudo, comumente, o solicitante é da própria gerência. Nesses casos, o solicitante ainda emite um relatório, composto basicamente de um parecer técnico sobre o objeto de estudo. O formato desse relatório irá depender das partes interessadas e da complexidade do projeto. Pode ser em inglês ou português, um simples e-mail ou um relatório de dezenas de páginas, por exemplo.

Em relação ao grau de maturidade em gerenciamento de projeto, foi observado que os questionários dos avaliadores resultaram em médias, por aspectos, bem diferentes. Os intervalos de confiança das médias, figura 2, mostraram que os limites inferior e superior resultariam em níveis de maturidade diferentes. Nesse caso, em que o coeficiente de variação é muito alto, a mediana pode ser uma medida descritiva mais adequada para as amostras (STEVENSON, 1981) e por isso foi adotada para comparação da situação antes e depois do desenvolvimento dos processos do PMBOK. 


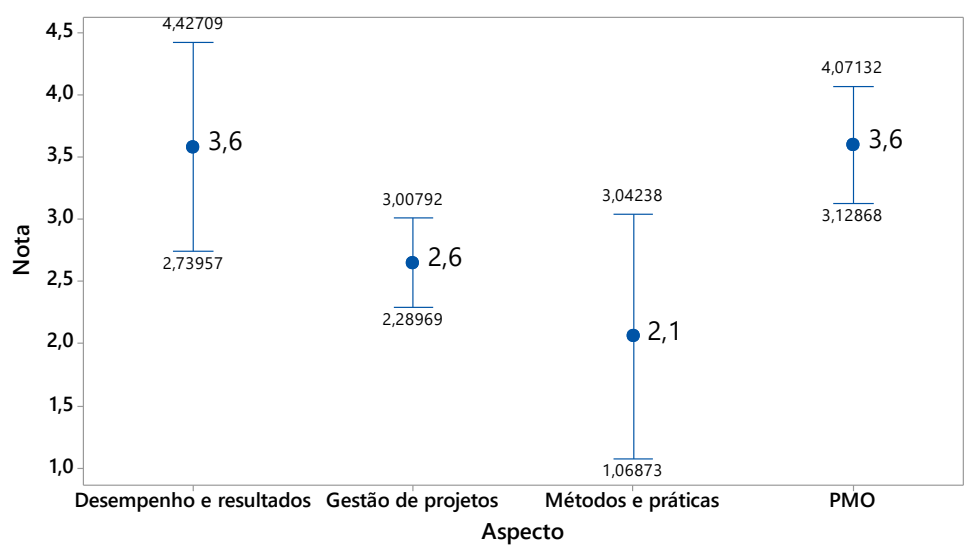

Figura 2. Média e intervalo de confiança média para o grau de maturidade em gerenciamento de projetos da área em 2015.

Os resultados dos questionários mostraram uma nítida evolução no grau de maturidade em gerenciamento de suas atividades, ou projetos, figura 3 . Essa evolução aumentou a nota de 1,3, ou nível conceitual, para 3,1 , ou nível padronizado.

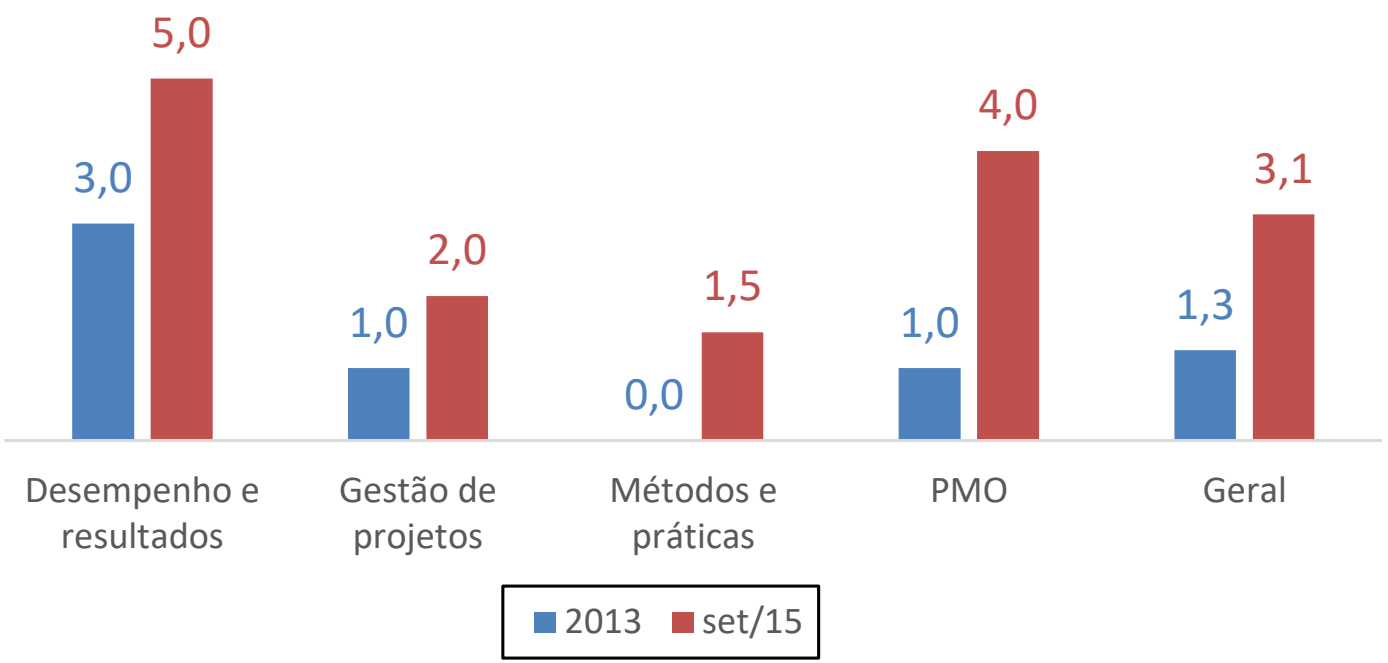

Figura 3. Evolução no grau de maturidade em gerenciamento de projetos da área.

Outra forma de se constatar a eficiência da aplicação de tais processos/ferramentas, é através do histórico do número de projetos executados pela gerência, conforme figura 4 . Verifica-se um aumento de $731 \%$ na quantidade de projetos realizados e devidamente documentados. Antes de 2012, esse controle não ocorria por ano e era pouco formalizado. 


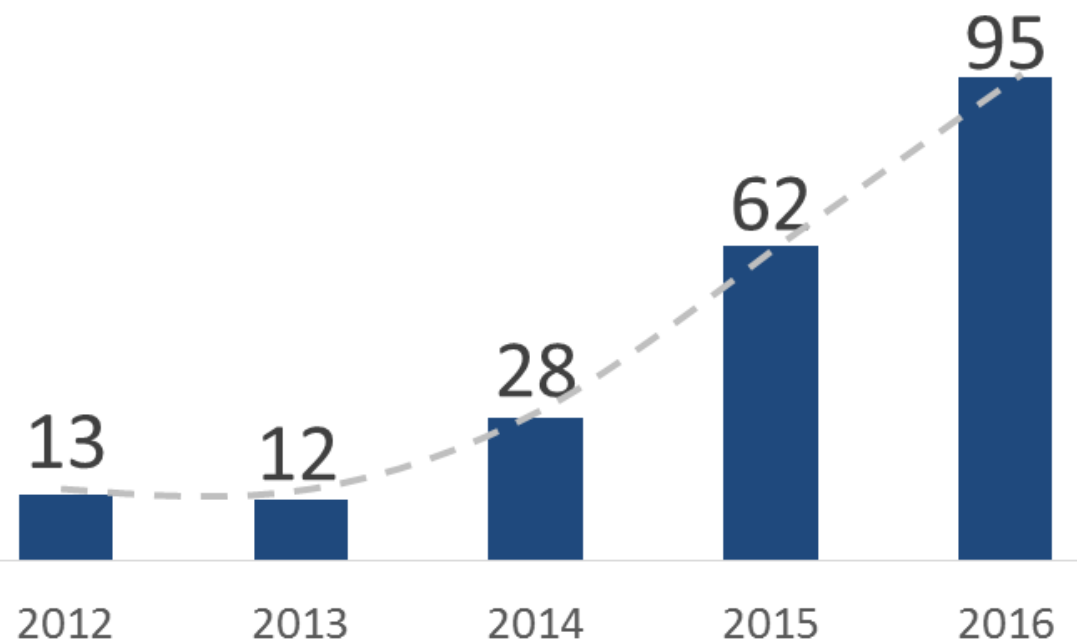

Figura 4. Quantidade anual de planos de trabalhos (ou projetos) realizados pela gerência de desenvolvimento de processos.

\section{CONCLUSÃo}

Conclui-se que os processos recomendados pelo PMBOK (PMI, 2013) são aplicáveis nas atividades da área de pesquisa e desenvolvimento de uma mineração de grande porte. A criação e aplicação de três ativos organizacionais padronizados (plano de trabalho, cronograma e planilha de resultados) para todas as atividades realizadas permitiu o desenvolvimento de grande parte dos processos considerados mais importantes. A aplicação de tais processos aumentou o sucesso das atividades através da melhoria no gerenciamento das mesmas. Essa melhoria no sucesso pode ser evidenciada através do amadurecimento da área em gestão de projetos e pelo aumento de atividades realizadas anualmente.

\section{Agradecimentos}

Agradecimento aos demais colegas de gerência pela inestimável ajuda e empenho em sugerir melhorias e colocar as ações em prática.

\section{REFERÊNCIAS}

1 BENZER, H.; DÜNDAR, H.; ALTUN, O.; TAVARES, L.M.M.; POWELL, M.; MAZZINGHY, D.B.; RUSSO, J.F.C.(2017) HPGR simulation from piston-die tests with an itabirite ore. REM - International Engineering Journal, v.70, p.99-107.

2 CRUZ, M.R. (2010) Flotação fracionada: aumento do rendimento da flotação através da divisão da alimentação em duas frações granulométricas, Dissertação de mestrado apresentada ao Curso de Pós-Graduação em Engenharia Metalúrgica e de Minas Dissertação de Mestrado

3 ESTEVES, P.; STOPA, M.M.; GALERY, R.; MAZZINGHY, D.B.; RUSSO, J.F.C. (2016) Relação entre potência elétrica e enchimento de carga moedora de moinhos verticais. Tecnologia em Metalurgia, Materiais e Mineração, v.13, p.288-294. 
4 MAZZINGHY, D.B.; LICHTER, J.; SCHNEIDER, C.L.; GALÉRY, R.; RUSSO, J.F.C. (2017) Vertical stirred mill scale-up and simulation: Model validation by industrial samplings results. Minerals Engineering, v.103, p.127-133.

5 MAZZINGHY, D.B.; RUSSO, J.F.C.; TAVARES, L.M. (2013) The investigation of comminution of itabirite iron ores using a pilot-scale HPGR. In: 13th European Symposium on Comminution \& Classification, v.1. p.47-50.

6 MAZZINGHY, D.B.; TURRER, H.D.G.; RUSSO, J.F.C; TAVARES, L.M. (2014) Influence of the mineralogical structure in grinding specific energy consumption of itabirate iron ores. In: Anais do Comminution 2014, 9p.

7 NETO, J.C.A.S. (2011) Avaliação de maturidade no gerenciamento de projetos em uma empresa de mineração em Minas Gerais. Dissertação de Mestrado em Administração apresentada à faculdade de Ciência Empresariais da Universidade FUMEC, 106p.

8 PMI - Project Management Institute (2013) Um Guia do Conhecimento em Gerenciamento de Projetos - Guia PMBOK®, Quinta Edição, 567p.

9 STEVENSON, W.J. (1981) Estatística aplicada à administração. Harper \& Row do Brasil, p.23

10 TURRER, H.D.G., MENEZES, K.B., MAGALHÃES, C.R., MAZZINGHY, D.B. (2013a) Comportamento e efeito do TiO2 no tratamento do minério de ferro. In: XXV Encontro Nacional de Tratamento de Minérios e Metalurgia Extrativa \& VIII Meeting of the Southern, p.487-495.

11 TURRER, H.D.G.; MAZZINGHY, D.B.; NASCIMENTO, C.M.; RUSSO, J.F.C.; DIAS, G.S. (2015) Desempenho no processamento mineral de dois tipos de itabiritos da Serra do Sapo. In: 45 Seminário de Redução de Minério de Ferro e Matérias-primas, 16을 Simpósio Brasileiro de Minério de Ferro e 3ํㅗㅇㅡósio Brasileiro de Aglomeração de Minério de Ferro, ABM, 9p.

12 TURRER, H.D.G.; MENEZES, K.B.; GONZAGA, F.H.P.; SILVA, J.C.; ALMEIDA, J.A.; MAGALHÃES, C.R.; RUSSO, J.F.C; PIMENTEL, D.A.; MAZZINGHY, D.B. (2013b) Concentração magnética para recuperação do ferro contido no rejeito da flotação. In: Anais do $43^{\circ}$ Seminário de Redução de Minério de Ferro e Matérias-primas, 14ํㅡㄴ Simpósio Brasileiro de Minério de Ferro e 1 Simpósio Brasileiro de Aglomeração de Minério de Ferro, ABM, 9p. 